\title{
A Primary Adenosquamous Carcinoma of the Stomach: Report of Two Cases
}

Melin GEÇER ${ }^{1}$, Nurcan ÜNVER ${ }^{1}$, Mustafa HASBAHÇECí3 ${ }^{3}$, Zuhal GÜCİN ${ }^{1}$, Zeynep TOSUNER'1 Ezgi ÇOBAN², Adem AKÇAKAYA ${ }^{3}$, Sema ARICI ${ }^{1}$

${ }^{1}$ Clinic of Pathology, Bezmialem Vakıf University Hospital, İstanbul, Turkey

${ }^{2}$ Clinic of Onkology, Bezmialem Vakıf University Hospital, İstanbul, Turkey

${ }^{3}$ Clinic of General Surgery, Bezmialem Vakıf University Hospital, İstanbul, Turkey

\section{ABSTRACT}

Primary gastric adenosquamous carcinomas are extremely rare tumors. A gradual transition between the malignant glandular and squamous components of the tumor is seen on performing a histopathological evaluation. These tumors are known to have poorer clinical outcomes than conventional adenocarcinomas. We aimed to present two cases of primary adenosquamous carcinomas that were diagnosed at the later stage in our institute.

Keywords: Adenosquamous carcinomas, stomach, poor prognosis

\section{Introduction}

Gastric adenosquamous carcinomas are mixed neoplasms containing gland-like and squamous areas. These rare tumors constitute less than $0.5 \%$ of all gastric neoplasms (1). It is more common in the sixth decade of life among Asians. The male:female ratio is 4:1. Primary gastric adenosquamous carcinomas are more aggressive than adenocarcinomas and have worse prognosis $(2,3)$.

Intestinal-type adenocarcinomas may exhibit squamous differentiation, and it is required that the squamous component should constitute more than $25 \%$ of the tumor for the diagnosis of adenosquamous carcinoma $(2,4)$. Since most of the rare carcinomas are published as case reports in literature, detailed information about their clinical course and prognosis is not available (5). In this report, we aimed to present two patients diagnosed with primary gastric adenosquamous carcinoma.

\section{Case Report}

\section{Case 1}

A 71-year-old male patient with abdominal pain, vomiting, and loss of appetite was examined due to microcytic hypochromic anemia, and a tumoral mass was detected in the antrumcorpus of the stomach. Surgical treatment was planned since the endoscopic biopsy results indicated low-differentiated adenocarcinoma in the squamous areas. A mass sized approximately $7 \mathrm{~cm}$ in diameter and located in the corpus-antrum of the stomach was detected as the operative finding. Distal subtotal gastrectomy, D2 lymph node dissection, and Roux-en-Y gastrojejunostomy were performed. The patient was discharged on the sixth postoperative day without any problems. The formation of a $8 \times 7 \times 5 \mathrm{~cm}$ ulcero-vegetating tumor that was located in the lesser curvature of the corpus and antrum, and was $5 \mathrm{~cm}$ from the proximal surgical margin and $5.5 \mathrm{~cm}$ from the distal surgical margin was macroscopically observed in the gastrectomy material. The tumor sections were gray-white and hemorrhagic. A microscopic examination of the tumor showed squamous carcinoma components

Cite this article as: Geçer M, Ünver N, Hasbahhçeci M, Gücin Z, Tosuner Z, Çoban E, et al. A Primary Adenosquamous Carcinoma of the Stomach: Report of Two Cases. Bezmialem Science 2017;5: 135-7. 
$(70 \%)$ in the large areas and adenocarcinoma components in the narrower areas (30\%; Figure 1). Both components had slight differentiation. Immunohistochemically, positive expressions of p63 in the squamous areas of the tumor and of CK7 and CEA in the adenocarcinoma component were observed (Figure 2). The presence of mucin was detected in the areas of adenocarcinoma using periodic acid-Schiff (PAS) and mucicarmine staining (Figure 1). There was lymphovascular invasion in the tumor invading the subserosa (T3). Among the total 33 lymph nodes detected, 5 metastatic lymph nodes (N2) were noted in the lesser curvature.

There was only adenocarcinoma in lymph node metastases, and no squamous component was found. Atypical or metastatic cells were not detected in the sample of intra-abdominal washing fluid. The patient was assessed as stage IIIA according
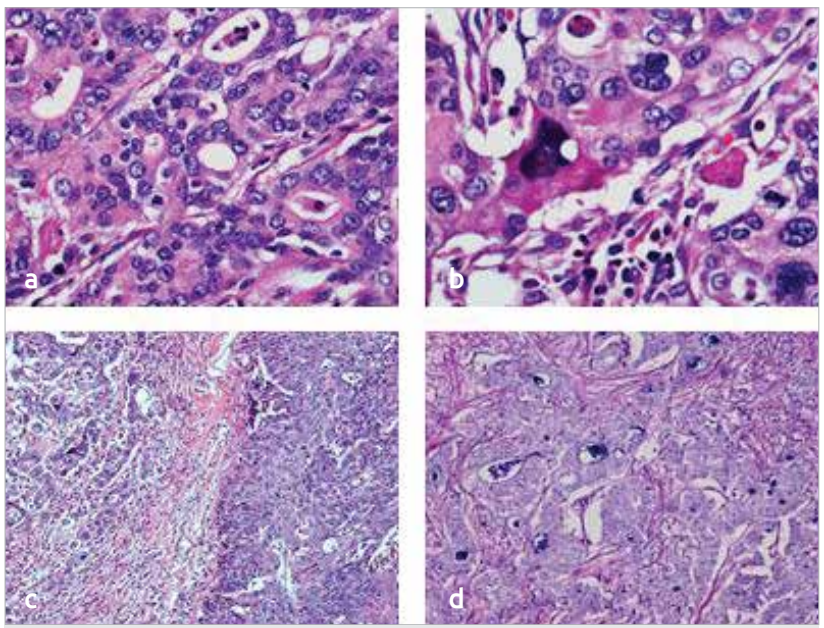

Figure 1. a-d. (a) Adenocarcinoma component of the tumor (HEX400), (b) squamous cell carcinoma component (HEX400), (c) adenosquamous areas (HEX200), and (d) PAS (+) mucin (X400) in adenocarcinoma areas
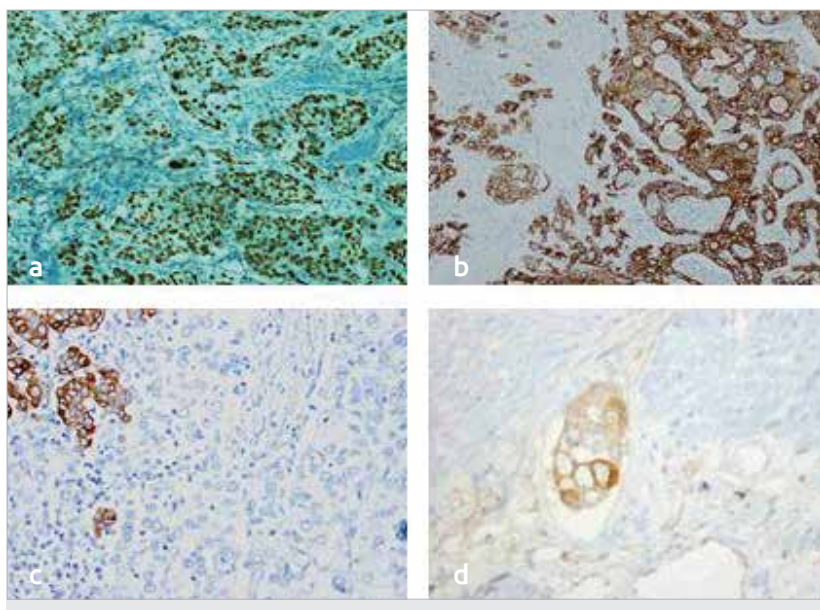

Figure 2. a-d. (a) Positive staining with p63 in the squamous carcinoma areas (X200), (b) positive staining with CK7 in the adenocarcinoma areas (X200), (c) CK7 negativity in the squamous carcinoma and CK7 positivity in adenocarcinoma in the same area (X200) (d) CEA expression in adenocarcinoma (X200) to the seventh version of the current American Joint Committee for Cancer (AJCC) criteria and was included in the adjuvant chemotherapy (oral capecitabine-intravenous cisplatin) protocol. The patient who was receiving chemotherapy had no additional problems at the postoperative fifth month control.

\section{Case 2}

A mass beginning from the stomach corpus and extending to the lesser curvature was found in the examinations of a 71-year-old male patient with the complaint of not being able to receive solid food. Surgical treatment was planned for the patient whose endoscopic biopsy was evaluated as mucinous adenocarcinoma. A $10-\mathrm{cm}$ mass that advanced to the antrum beginning from the stomach corpus was detected in the operation. Total gastrectomy, D2 lymph node dissection, and Roux en Y esophagojejunostomy were performed. The patient was discharged on the sixth day without any problems in the postoperative period. An $11.5 \times 10.5 \times 2.2$-cm ulcerovegetating tumor that was located in the lesser curvature of the corpus and antrum and was $6 \mathrm{~cm}$ from the proximal surgical margin and $1.5 \mathrm{~cm}$ from the distal surgical margin was macroscopically observed in the gastrectomy material. A total of 38 lymph nodes, the largest of which was $25 \mathrm{~mm}$, were detected in the specimen. Microscopic examinations of the gastric tumor showed squamous components (60\%) and adeno components $(40 \%)$ in the large areas. Both components had slight differentiation. Immunohistochemically, positive expressions of p63 and CK 5/6 in the squamous areas of the tumor and of CK7 and CDX2 in the adeno component were observed. Staining was observed using PAS and mucicarmine in the mucin existing within the adeno component. The tumor was invading the subserosa (T3), and there were lymphovascular, perineural, and plexus invasions. The microscopic examination of the lymph nodes revealed adenosquamous lymph node metastasis (N3) in a total of 7 lymph nodes. No atypical or metastatic cells were detected in the sample of intra-abdominal washing fluid. With these findings, the patient was evaluated as stage IIIB according to the seventh version of the AJCC criteria for stomach cancer staging and was included in the adjuvant chemotherapy protocol (oral capecitabineintravenous cisplatin). The patient had no problems in the postoperative first year control.

\section{Discussion}

Adenosquamous carcinomas are a particular and rare type of gastric carcinomas and constitute less than $0.5 \%$ of all gastric cancers (6). The tumors can be seen in each region of the stomach, are highly aggressive tumors, and are usually in the advanced stage when diagnosed. They are rarely diagnosed as early stomach cancers, often metastasize to the liver, and lymphovascular invasion is frequent. The 5-year survival is approximately $10 \%$ and the average survival time is 12 months (7). Consistent with literature, both patients presented in this report were in advanced age and were males, with stages IIIA and IIIB, respectively. While distal subtotal gastrectomy was 
sufficient for the distal tumor in one patient, total gastrectomy was required for the tumor located in the corpus and antrum in the other patient.

The etiology of adenosquamous carcinoma is unclear. There are several hypotheses for the formation of gastric carcinomas of this type:

1- Metaplastic transformation of adenocarcinoma

2- Carcinoma of the metaplastic squamous epithelium in the gastric mucosa

3- Carcinoma of the ectopic squamous epithelium in the gastric mucosa

4- Differentiation of endothelial cells to squamous cells

5- Differentiation of stem cells to both epithelia (8)

However, the pathogenic mechanisms are not clearly known due to the low number of cases.

Adenosquamous carcinoma is a combination of adenocarcinoma and squamous cell carcinoma, and there is a transition between two tumors.

Although the tumor has two separate components, the biological behavior is determined by adenocarcinoma. Symptoms are not different from those of adenocarcinoma.

In both cases, adenocarcinoma and squamous cell carcinoma components and a gradual transition between these two components were observed (Figure 1). Although squamous areas are predominant in both tumors, interestingly, adenocarcinoma component was found in the biopsies of both cases. Although adenocarcinoma component determines the biologic behavior of adenosquamous carcinoma, another characteristic of these two cases is that lymph node metastases are seen as adenocarcinoma in the first case and as adenosquamous carcinoma in the second case. In the seven-case series by Chen et al (1), while lymph node metastasis developed only from adenocarcinoma in six cases, only squamous carcinoma was detected in one case. In another series of 13 cases, adenocarcinoma was detected in eight of 12 patients with $\mathrm{N}+$ and both components, and only squamous components were detected in each two cases (2). Since no evident-based knowledge is available in literature on the amount of squamous component in the lymph node metastases and its effect on the prognosis, it may be possible to assess the reason for this difference in a larger series.

\section{Conclusion}

The two patients presented in this report were still alive at the end of 5-and 12-month follow-ups, and no evidence of recurrence of the disease or metastasis was found. Adjuvant chemotherapy was administered because both cases were stage III. However, a standard chemotherapy protocol for adenosquamous stomach carcinomas remains undefined (2, 8). Thus, oral capecitabineintravenous infusion with cisplatin chemotherapy was administered considering the age and general condition of the patients. Although the follow-up time is not sufficient to comment on the clinical course of the disease, a relatively poor prognosis may be expected due to the advanced stage gastric cancer found during the diagnosis.

Informed Consent: Written informed, consent was obtained from patient who participated in this study.

Peer-review: Externally peer-reviewed.

Author Contributions: Concept - Z.G., M.G.; Design - Z.T., M.G.; Supervision - M.H., Z.G.; Funding - N.Ü., M.G.; Materials - Z.G., M.G.; Data Collection and/or Processing - M.G., N.Ü.; Analysis and/or Interpretation - M.G.; Literature Review - M.G.; Writing - M.G., M.H.; Critical Review - Z.G., Z.T., M.G.

Conflict of Interest: No conflict of interest was declared by the authors.

Financial Disclosure: The authors declared that this study has received no financial support.

\section{References}

1. Chen H, Shen C, Yin R, Yin Y, Chen J, Han L, et al. Clinicopathological characteristics, diagnosis, treatment, and outcomes of primary gastric adenosquamous carcinoma. World J Surg Oncol 2015; 13: 136. [CrossRef]

2. Chen YY, Li AF, Huang KH, Lan YT, Chen MH, Chao Y, et al. Adenosquamous carcinoma of the stomach and review of the literature. Pathol Oncol Res 2015; 21: 547-51. [CrossRef]

3. Yuan-Tzu Lan, Kuo-Hung Huang, Chien-An Liu, Ling-Chen Tai, Ming-Huang Chen, Yee Chao, et al. A Nation-Wide Cancer RegistryBased Study of Adenosquamous Carcinoma in Taiwan. PLoS One 2015; 10(10). [CrossRef]

4. Saito S, Hosoya Y, Morishima K, Ui T, Haruta H, Kurashina K, Meguro $\mathrm{Y}$, et al. A clinicopathological and immunohistochemical study ofgastric cancer with squamous cell carcinoma components: A clinically aggressive tumor. J Dig Dis 2012; 13: 407-13. [CrossRef]

5. Ajoodhea H, Zhang RC, Xu XW, Jin WW, Chen K, He YT, et al. Fever as a first manifestation of advanced gastric adenosquamous carcinoma: A case report. World J Gastroenterol 2014; 20: 10193-201. [CrossRef]

6. Rinkesh Kumar Bansal, Praveen Sharma, Ramneet Kaur, Anil Arora. Primary gastric adenosquamous carcinoma in an Indian male. 2013; 56: 416-8.

7. Kadowaki S, Yatabe Y, Nitta S, Ito Y, Muro K. Durable response of human epidermal growth factor receptor-2-positive gastric adenosquamous carcinoma to trastuzumab-based chemotherapy. Case Rep Oncol 2014; 7: 210-6. [CrossRef]

8. Shirahige A, Suzuki H, Oda I, Sekiguchi M, Mori G, Abe S, et al. Fatal submucosal invasive gastric adenosquamous carcinoma detected at surveillance after gastric endoscopic submucosal dissection. World J Gastroenterol 2015; 21: 4385-90. [CrossRef] 